\title{
Local application of antimycotics in mucormycosis cerebri: a case report
}

\author{
I Mohsenipour, M Schirmer, R Frank, F Geisen, E Semenitz, D zur Nedden, \\ G Konwalinka
}

\begin{abstract}
A 65 year old man was found to have mucormycosis cerebri during immunosuppression after treatment of hairy cell leukaemia with 2-chlorodeoxyadenosine. Although mucormycosis cerebri has a poor prognosis, the patient survived after systemic administration of high dose amphotericin $B$, extensive excision of the abscess, and additional local application of amphotericin $B$ with the help of an absorbable gelatin sponge.
\end{abstract}

(† Neurol Neurosurg Psychiatry 1996;61:521-522)

Keywords: immunosuppression; mucormycosis cerebri; absorbable gelatin sponge; 2-chlorodeoxyadenosine

Cerebral abscess caused by Mucoraceae is rare and has a poor prognostic outcome. Only a few cases of successfully treated primary mucor cerebral abscess have been reported. ${ }^{1-5}$ Such abscesses are often associated with diabetes mellitus, intravenous drug misuse, craniofacial infections, or immunosuppression, ${ }^{6}$ with immunocompromised patients having an especially increased risk of opportunistic infections. Up to now, implanted reservoirs have been used to ensure high concentrations of antibiotics in CSF.?

We describe the successful application of an absorbable gelatin sponge in a patient who had invasive cerebral mucormycosis (Rhizomucor) during the leucopenic phase after treatment of hairy cell leukaemia with 2 -chlorodeoxyadenosine $(2-\mathrm{CdA})$.

\section{Patient's history and study}

A 65 year old man with a history of hairy cell leukaemia and severe pneumonia was transferred to our hospital from a foreign clinic for treatment of hairy cell leukaemia with 2-CdA, which can induce complete remission of hairy cell leukemia in more than $80 \%$ of patients. ${ }^{8}$ On admission his white cell count was $2700 / \mathrm{mm}^{3}$. Bone marrow biopsy disclosed infiltration with $80 \%$ hairy cells. Other laboratory values were normal, including creatinine, uric acid, and lactate dehydrogenase concentrations. The patient was treated with a daily infusion of $0.1 \mathrm{mg} / \mathrm{kg} 2-\mathrm{CdA}$ (Leustatin $^{\mathrm{TM}}$, Cilag, Austria) for seven days. Leucocyte numbers fell to below 50 cells $/ \mathrm{mm}^{3}$ during the next few days.

On day 12 after beginning treatment with 2$\mathrm{CdA}$, his temperature rose to $39^{\circ} \mathrm{C}$. The general condition of the patient deteriorated dramatically, two syncopes occurred, and hemiparesis of the left side developed. The patient had two attacks of grand mal epilepsy. Despite immediate initiation of antimycotic therapy with amphotericin B up to a dosage of $1.0 \mathrm{mg} / \mathrm{kg}$, the cerebral tumour grew as evidenced by CT (fig 1). Therefore craniotomy with aspiration of the abscess was performed. Histological preparations stained with haematoxylin-eosin and Grocott-Gomori-methenamine-silver allowed the definite diagnosis of invasive fungal infection with Rhizomucor. In culture, the organism was sensitive to amphotericin B only. Therefore, the daily dosage of amphotericin B was increased to $4.0 \mathrm{mg} / \mathrm{kg}$ by using the liposomal form (AmBisome ${ }^{\mathrm{TM}}$, Vestar, USA). This high dose was well tolerated, but hemiparesis of the left side became more severe and the patient became disoriented.

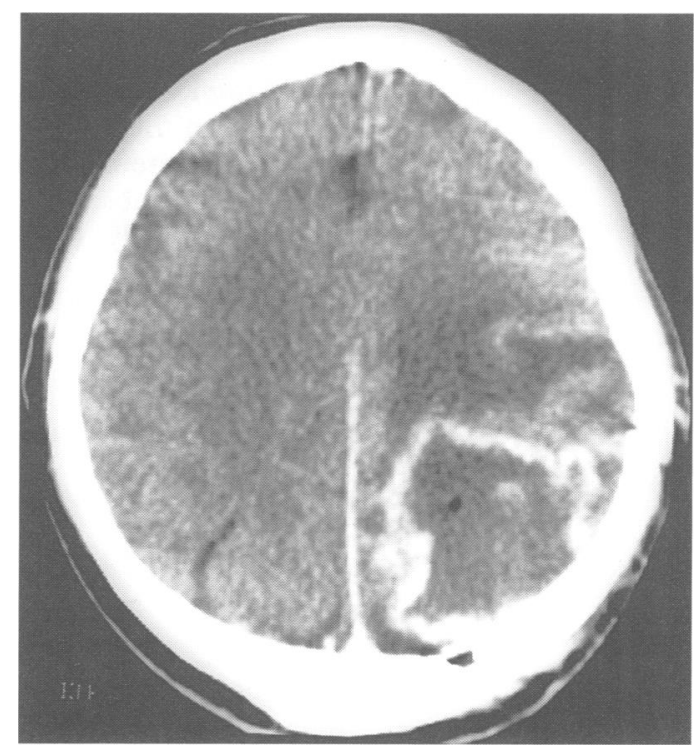

Computed tomography obtained before surgical intervention, showing the cerebral abscess caused by Mucoraceae in the immunocompromised patient. 
Eighteen days after the first craniotomy, resection of the abscess, including removal of the abscess membrane was performed. The cavity was packed with an absorbable gelatin sponge (Spongostan ${ }^{\mathrm{TM}}$, Ferrosan, Denmark) $3 \times 2 \times 2 \mathrm{~cm}$ in size, saturated with $30 \mathrm{mg}$ of amphotericin B $\left(2.5 \mathrm{mg} / \mathrm{cm}^{3}\right.$ of the cavity).

Under prolonged continuation of systemic antimycotics, and antiepileptic and anti-oedematous therapy, the patient gradually recovered. Brain CT showed cystic alterations with little enhancement of contrasting agents and minimal perifocal oedema. To diminish secondary haematological dissemination systemic treatment with a normal dosage of amphotericin $\mathbf{B}$ was given during the next seven weeks. The patient survived with subsequent complete remission of hairy cell leukaemia. It is now three years since the occurrence of mucor cerebral abscess during the neutropenic phase after 2-CdA treatment and recovery followed with only minor residual neurological deficits.

\section{Discussion}

After surgical debridement, we placed a gelatine sponge with amphotericin B in the cavity of the cerebral abscess caused by mucormycosis. Implanted reservoirs are often complicated by secondary bacterial infections, displacement of the ventricular catheter, or obstruction of the system, ${ }^{7}$ whereas a sponge is absorbed after four to six weeks. Thus no further intervention was necessary.

Survival of an immunocompromised heavily neutropenic patient with primary mucormycosis cerebri is very rare. $\mathrm{Up}$ to now, all cases of candidasepsis or aspergilloma of the CNS after treatment with 2-CdA have been reported to end with fatal outcome. ${ }^{9}$ This can be explained by the continuing heavy decrease of the helper $T$ cell counts for more than six months in patients with hairy cell leukaemia after treatment with $2-\mathrm{CdA},{ }^{10}$ resulting in a severely deranged immunocompromised status. In our immunocompromised patient, too, maximal systemic application of amphotericin B (1 mg/kg of normal amphotericin B and $4 \mathrm{mg} / \mathrm{kg}$ of the liposomal form) remained ineffective despite comitant craniotomy with aspiration of the abscess. The cerebral abscess (fig 1) had already caused serious neurological deficits.
As only $2 \%-4 \%$ of systemically administered amphotericin B passes the blood-brain barrier in adults, ${ }^{11}$ in our patient, with a body weight of $75 \mathrm{~kg}$, a maximum of $12 \mathrm{mg}$ of systemically applied amphotericin B could be expected to pass the blood-brain barrier. This resulted in a concentration of $24 \mu \mathrm{g} / \mathrm{ml}$ in the CSF, which is not high enough for a fungicidal effect of amphotericin B. On the other hand we did not find any reports about the highest concentration tolerated in the CSF. The exact clearance of amphotericin B in the CSF is also unknown. We assumed a daily production of $500 \mathrm{ml} \mathrm{CSF}$. To ensure a fungicidal concentration of $50 \mu \mathrm{g} / \mathrm{ml}$ during a prolonged release of amphotericin B by the gelatin sponge we considered $30 \mathrm{mg}$ of amphotericin B not to be toxic if applied locally in addition to the systemic application of amphotericin B. This estimation proved realistic and our patient did not have chills, nausea, or headache, and had no other major side effects.

In view of the positive outcome in this immunocompromised patient, additional local installation of amphotericin $\mathbf{B}$ using a resorbable gelatin sponge might prove helpful in similar cases after treatment with $2-\mathrm{CdA}$.

We thank our colleagues at the Kantonsspital Aarau, Switzerland, for performing the rehabilitation programme of the patient. This work was supported by the Austrian Research Council (FWF) P 10132 med (GK).

1 Fong KM, Seneviratne EM, McCormack JG. Mucor cerebral abscess associated with intravenous drug abuse. Aust $N Z \nsucceq$ Med 1990;20:74-7.

2 Morrison VA, McGlave PB. Mucormycosis in the BMT population. Bone Marrow Transplant 1993;11:383-88.

3 Rao VR, Pillai SM, Mathews G, Radhakrishnan VV. Cerebral mucormycosis-a case report. Neuroradiology 1978;15:291-93.

4 Riefler J, Batbouta J, Uphoff DF. Case reports Rhizopus brain abscess: report of a case and review of the literature. Mil Med 1991;156:497-99.

5 Woods KF, Hanna BJ. Brain stem mucormycosis in a narcotic addict with eventual recovery. $\mathrm{Am} \mathcal{F} \mathrm{Med} 1986$; 80:126-28.

6 Lehrer RI, Howard DH, Sypherd PS, Edwards JE, Segal GP, Winston DJ. Mucormycosis. Ann Intern Med 1980; 93:93.

7 Reynolds JEF, Parfitt K. Martindale, the extra pharmacopoeia. London: The Pharmaceutical Press, 1989: 417-20.

8 Piro LD, Carrera CJ, Carson DA, Beutler E. Lasting remissions in hairy-cell leukemia induced by a single infusion sions in hairy-cell leukemia induced by a single infusion of 2-chlor

9 Juliusson G, Liliemark J. Rapid recovery from cytopenia in hairy-cell leukemia after treatment with 2-chloro-2'deoxyadenosine $(\mathrm{CdA})$ : relation to opportunistic infections. Blood 1992;79:888-94.

10 Beutler E. Cladribine (2-chlorodeoxyadenosine). Lancet 1992;340:952-6.

11 Benson JM, Nahata MC. Clinical use of systemic antifungal agents. Clin Pharm 1988;7:424-38. 